\title{
Desde la displasia hasta el cáncer de la vesícula biliar
}

\author{
Iván Roa $E^{1,2}$, Sergio Muñoz $N^{3}$, G ilda Ibacache $S^{2}$, \\ Xabier de Aretxabala U4.
}

\section{Natural history of gallbladder cancer. Analysis of biopsy specimens}

Background: There is scarcity of knowledge about the development of gallbladder cancer. Aim: To study the features of development and progression of gallbladder cancer. Material and methods: Review of histopathological studies of gallbladder obtained in 25,971 cholecytectomies performed in patients aged $45 \pm 16$ years, $79 \%$ females, between 1993 and 2004. Among these, 210 had a dysplasia not associated to cancer and 1,039 had a gallbladder cancer. Clinical and morphological parameters of preneoplastic and neoplastic lesions were analyzed. Ninety five percent of patients were followed. Results: All cases of dysplasia were incidental findings. Metaplasia, dysplasia and carcinoma in situ were present in the adjacent mucosa in $66 \%, 81 \%$ y $69 \%$ of gallbladder carcinomas, respectively. Twenty five percent of gallbladders studied were carcinomas (mucous carcinoma in $18 \%$ and muscular carcinoma in 7\%). Ninety two percent of cases had chronic inflammation in the gallbladder wall. Seventy two percent of mucous carcinomas were not detected macroscopically. Five years survival of mucous carcinoma was $92 \%$. There was an association between the intensity of the lesion and the age of the patients. The age difference between chronic cholecystitis and gallbladder cancer was 11 years for women and nine for men. Conclusions: From a morphological standpoint, the period in which a dysplasia becomes a carcinoma is approximately 10 years (Rev Méd Chile 2009; 137: 873-80).

(Key w ords: Cholecystectomy; Gallbladder neoplasms; Metaplasia)

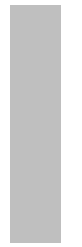

Recibido el 22 de diciembre, 2008. Aprobado el 8 de mayo, 2009.

Trabajo financiado por Proyectos Fondecyt 1060375 y Bicentenario PBCT6.

${ }^{1}$ Servicio de Anatomía Patológica, Clínica Alemana de Santiago. Facultad de Medicina, Universidad del Desarrollo. Santiago de Chile. ${ }^{2}$ Facultad de Medicina, Universidad de la Frontera. Temuco, Chile. ${ }^{3}$ Centro de Investigación y Gestión en Salud, Facultad Medicina, Universidad de la Frontera, Temuco, Chile. ${ }^{4}$ Departamento de Cirugía, Clínica Alemana de Santiago. Chile.

$\mathrm{C}$ hile es uno de los países con mayor frecuencia de cáncer de la vesícula biliar (CVB) en el mundo en ambos sexos ${ }^{1-3}$. En la mujer chilena, representa la primera causa de muerte por tumo-

Correspondencia a: Dr. Iván Roa E. Avenida Vitacura 5951, Vitacura. Santiago, Chile. E mail: iroa@alemana.cl res malignos desde 1985, con una proporción de 4:1 respecto de los hombres 4,5 .

Aun cuando se reconocen múltiples factores de riesgo del CVB, el conocimiento acerca de esta neoplasia es significativamente menor en comparación con otros tumores malignos como pulmón, mama o colon ${ }^{6}$, probablemente debido a que esta neoplasia es poco frecuente en la mayoría de los países desarrollados, a excepción de algunas áreas 
del Japón ${ }^{7,8}$. El diagnóstico de CVB se establece en la mayoría de los casos en estadios avanzados de la enfermedad, por lo cual, el estudio de los mecanismos que participan en su génesis y desarrollo en las etapas tempranas resulta particularmente difícil ${ }^{9,10}$.

Entre los factores de riesgo más importantes de CVB se encuentran los cálculos vesiculares ${ }^{11-13}$, el género, edad, obesidad, paridad ${ }^{14,15}$, tasa de colecistectomías $^{16,17}$, inflamación crónica ${ }^{6}$, los adenomas vesiculares ${ }^{18,19}$ y la unión anómala pancreático-biliar ${ }^{20,21}$, entre otros.

Los pacientes con CVB en su gran mayoría son asintomáticos hasta estadios avanzados de la enfermedad. La litiasis sintomática y la inflamación crónica de la vesícula biliar son las manifestaciones clínicas más frecuentes asociadas al $\mathrm{CVB}^{2,22}$. Este hecho determina que en Chile, miles de pacientes anualmente sean colecistectomiza$\operatorname{dos}^{1,5,23}$, es en este grupo de pacientes en los que en forma incidental se diagnostican tanto las lesiones precursoras como el $\mathrm{CVB}^{24}$.

La displasia epitelial y los adenomas son lesiones reconocidas como lesiones preneoplásicas en la mucosa de la vesícula biliar, cada una de ellas representante de modelos carcinogenéticos diferentes ${ }^{10,19,25,26}$. Evidencias de carácter morfológico y genético-molecular sustentan la validez de ambos modelos ${ }^{27-29}$.

Los criterios histológicos diagnósticos de la displasia asociada o no al CVB, son poco reproducibles $^{25,30}$ y han sido reportadas en nuestro país con frecuencias extremadamente variables ${ }^{10,31}$. La displasia de la mucosa vesicular no asociada a la presencia de un cáncer ha sido reportada en porcentajes que fluctúan entre $1 \%$ y $20 \% 10,30,32,33$. Las diferencias que pudiesen existir entre la displasia relacionada a un CVB y la observada en forma aislada no asociada a cáncer no han sido estudiadas, por lo cual, su teórica progresión es sólo una extrapolación de lo observado en la carcinogénesis en otros órganos.

Establecer la relación entre la displasia y el CVB representa una de las mayores dificultades producto de las características del órgano y la imposibilidad en el seguimiento de las lesiones displásicas en la vesícula biliar. No existe información respecto de la existencia de displasia epitelial en vesículas biliares sin litiasis o inflamación crónica a excepción de las observadas en algunas malformaciones de la unión pancreático-bi$\operatorname{liar}^{34,35}$

El objetivo de este trabajo es analizar aspectos relevantes de las lesiones consideradas como precursoras y las formas más precoces de cáncer vesicular intentando establecer la continuidad en el proceso carcinogenético en la mucosa vesicular.

\section{MATERIAL Y MÉTODO}

Casos. Se incluyen todas las piezas de colecistectomías procesadas histológicamente (25.971 casos) del Hospital de Temuco entre los años 1993 y 2004, en las que se diagnosticaron 1.039 CVB, 210 displasias no asociadas a cáncer de la vesícula biliar (0,8\%) y 45 adenomas (0,17\%).

Protocolo de procesamiento. El procesamiento de las piezas de colecistectomía fue realizado en forma uniforme de acuerdo a protocolo establecido en 1987 y reportado previamente ${ }^{36}$. Este protocolo resumidamente consiste en: apertura de la vesícula biliar, fijación y extensión de la pieza quirúrgica en planchas de parafina sólida, fotografía macroscópica (hasta 1995) o digitalización. Para el estudio histológico de rutina se seleccionaron 3 muestras representativas de cada uno de los segmentos de la vesícula biliar. En todos los casos con diagnóstico de displasia o cáncer, las piezas quirúrgicas fueron mapeadas en forma completa y sistematizada (Figura 1).

Clasificación histológica. Se utilizó la clasificación de la OMS y de la AJCC de $1977^{37}$ y posteriormente la de $2002^{38}$, dependiendo del periodo.

Displasia. Fue definida como alteración de la maduración del epitelio glandular con frecuente irregularidad nuclear, hipercromasia, pérdida de la polaridad nuclear, seudo estratificación, alteración de la relación núcleo/citoplasma o presencia de mitosis atípicas ${ }^{25,30}$.

Nivel de infiltración. Posterior al mapeo de la pieza quirúrgica se reconocieron, de acuerdo al nivel de infiltración tumoral de la pared vesicular, los carcinoma incipientes: carcinoma in situ (CIS), carcinoma mucoso (CMu) y los carcinomas musculares (CMp) y un grupo de carcinomas avanza- 
dos: carcinomas subserosos (CSs), carcinomas serosos (CSe) y metástasis (Me).

Seguimiento de pacientes. El 95\% de los pacientes tuvo seguimiento, el que fue realizado a través de: a) controles y seguimiento clínico de los pacientes, b) registro nacional de identificación.

Estadística. Se realizó mediante los programas Winstat 3.0 y Stata 7.0. Se realizó análisis de la varianza (ANOVA) y t-test para los promedios, chi cuadrado para tablas de contingencia y curvas de sobrevida actuarial de Kaplan-Meier con análisis de significación mediante Log-rank test (CoxMantel).

\section{Resultados}

Características generales del grupo estudiado. De un total de 25.971 pacientes incluidos en este estudio, 79\% correspondió a mujeres con un promedio de edad de 45,4 años ( $\mathrm{DE} \pm 15,9$ años) y $21 \%$ restante a hombres con un promedio de edad de 53,6 años ( $D E \pm 16,4$ años) ( $p<0,0001$ ). De los 1.536 pacientes con lesiones preneoplásicas y neoplásicas de la vesícula biliar, 210 (13,6\%) correspondieron a displasias no asociadas a cáncer de la vesícula biliar y 1.326 casos de cáncer de la vesícula biliar, cuya distribución por sexo y nivel de infiltración tumoral se muestran en la Tabla 1. En 337 casos los tumores correspondieron a carcinomas incipientes (26,2\%), de los cuales 210 casos (16\%) eran CMu y los restantes
127 a CMp. El 52\% de los tumores (692 casos) eran cánceres avanzados con compromiso de la serosa, metástasis locales o con infiltración de órganos adyacentes.

El promedio de edad de la serie total de los 25.971 pacientes fue de 47,1 años ( $\mathrm{DE} \pm 16,4$ años). En estos pacientes se realizaron los distintos diagnósticos histológicos sin otra selección que la determinada por la indicación quirúrgica antes mencionada. Se observó una diferencia de nueve años en los promedios de edad entre hombres y mujeres en el grupo con diagnóstico de patología vesicular no tumoral ( $p<0,0001)$. Las mujeres presentaron promedios de edad significativamente menores que los hombres en todos los grupos de lesiones tumorales y no tumorales (45,4 años versus 53,6 años) ( $p<0,0001)$.

Displasia no asociada a cáncer vesicular. En nuestros casos constituye una lesión poco frecuente cercana a $1 \%$ de las colecistectomías por litiasis. Durante el período de estudio se diagnosticaron 210 casos $(0,8 \%)$, de los cuales 188 correspondieron a mujeres con un promedio de edad de 50,9 años ( $D E \pm 15,8$ años) y los restantes 22 a hombres con un promedio de edad de 58,5 años ( $D E \pm 13,2$ años) ( $p=0,008)$. El promedio de edad de este grupo de pacientes fue mayor que el grupo de pacientes sólo con colecistitis crónica y menor que el promedio de edad de los pacientes con la forma más precoz de los CVB (CMu).

Todas las displasias no asociadas a cáncer fueron un hallazgo incidental en el examen rutinario, sin una expresión macroscópica que

Tabla 1. Promedios de edad de pacientes. D istribución por género y tipo de lesión

\begin{tabular}{|lrrrrrrrrrc|}
\hline \multicolumn{1}{l}{ Tipo lesión } & \multicolumn{3}{c}{ Mujer } & \multicolumn{4}{c}{ Hombre } & \multicolumn{5}{c|}{ Total } \\
C. crónica & Promedio & DS & N & Promedio & DS & N & Promedio & DS & p \\
Displasia & 19.244 & 44,7 & 15,7 & 5.191 & 53,1 & 16,4 & 24.435 & 46,5 & 16,2 & 0,000 \\
C. mucoso & 188 & 50,9 & 15,8 & 22 & 58,5 & 13,2 & 210 & 51,9 & 15,7 & 0,008 \\
C. muscular & 171 & 55,6 & 14,6 & 39 & 62,3 & 12,8 & 210 & 56,8 & 14,5 & 0,00007 \\
C. subseroso & 107 & 60,9 & 14,0 & 20 & 64,5 & 11,7 & 127 & 61,5 & 13,6 & 0,281 \\
C. seroso & 259 & 62,3 & 12,8 & 38 & 67,2 & 12,9 & 297 & 62,9 & 12,9 & 0,02 \\
Metástasis & 302 & 61,5 & 13,3 & 66 & 66,9 & 10,5 & 368 & 62,5 & 13,0 & 0,002 \\
Total & 257 & 61,8 & 12,5 & 67 & 68 & 11,2 & 324 & 63,1 & 12,5 & 0,0002 \\
& 20.528 & 45,4 & 15,9 & 5.443 & 53,6 & 16,4 & 25.971 & 47,1 & 16,4 & 0,0001 \\
\hline
\end{tabular}


Figura 1. Esquema de mapeo de pieza de colecistectomía en todos los casos de displasia o cáncer de la vesícula biliar.
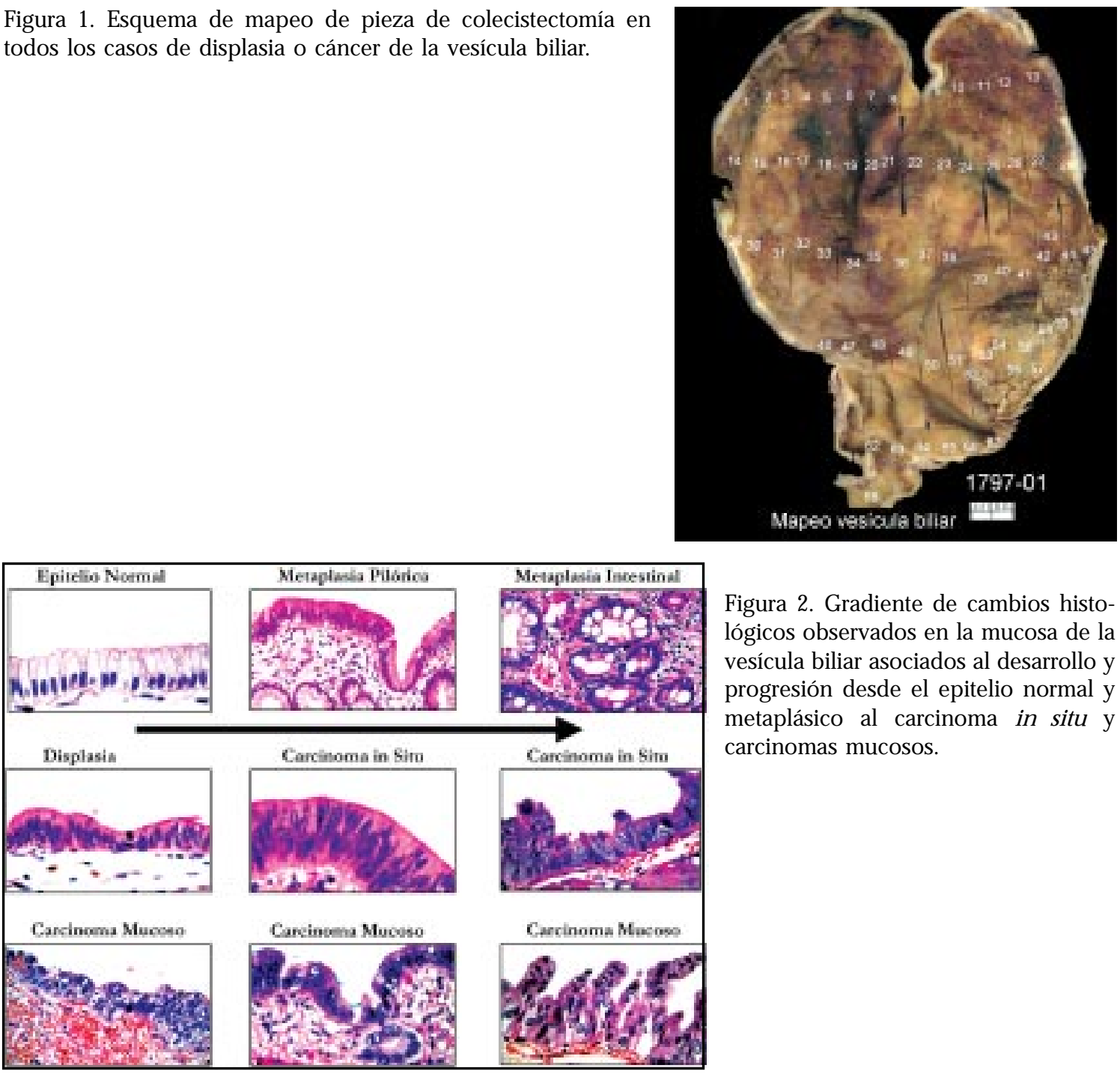

Figura 2. Gradiente de cambios histológicos observados en la mucosa de la vesícula biliar asociados al desarrollo y progresión desde el epitelio normal y metaplásico al carcinoma in situ $\mathrm{y}$ carcinomas mucosos.

Figura 3. Relación entre la edad de los pacientes e intensidad de las lesiones en hombres y mujeres.

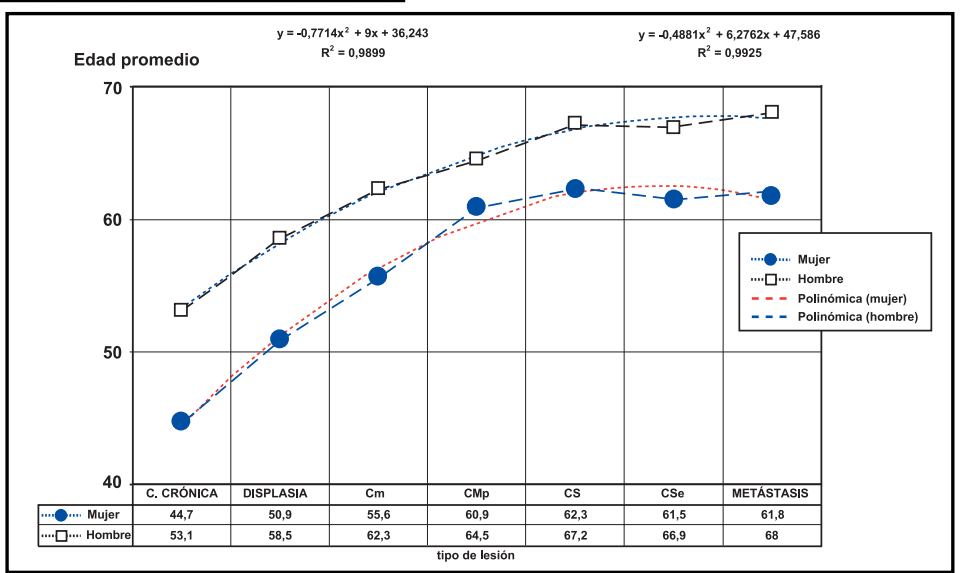


permitiese diferenciarlas de las alteraciones observadas en la mucosa vesicular crónicamente inflamada. La colesterolosis, se asoció negativamente a la presencia de displasia epitelial $(p<0,001)$ y la más frecuente alteración epitelial asociada a la displasia fue la metaplasia intestinal. Microscópicamente las alteraciones fueron más frecuentemente observadas en la superficie mucosa que en las estructuras glandulares.

Lesiones epiteliales asociadas al cáncer vesicular. La displasia epitelial ha sido considerada como la lesión preneoplásica a partir de la cual se desarrollaría el CIS que evolucionaría a un carcinoma infiltrante a través de un modelo morfológico continuo desde la displasia leve hasta la displasia intensa y CIS (Figura 2). De acuerdo a nuestros hallazgos, en la vesícula biliar con un carcinoma infiltrante la metaplasia, displasia y CIS están presentes en la mucosa adyacente al cáncer en $66 \%, 81,3 \%$ y $69 \%$, respectivamente. La localización de las lesiones intraepiteliales en relación al carcinoma infiltrante se observa en estrecha relación de vecindad en la mayoría de los casos (85\%), observándose focos de displasia o CIS aislados en menos de 10\% de los casos. Estos elementos apoyan fuertemente que la carcinogénesis vesicular se realizaría a través de la transformación del epitelio propio de la mucosa.

Carcinoma mucoso, la forma más precoz de cáncer vesicular. De un total de 1.326 cánceres vesiculares, $25 \%$ de los diagnosticados eran carcinomas incipientes (18\% CMu y $7 \% \mathrm{CMp})$. El promedio de edad de los 210 casos de $\mathrm{CMu}$ fue de 56,8 años ( $\mathrm{DE} \pm 14,5$ años). La paciente más joven tenía 16 años y la mayor tenía 92 años. En sólo 3 casos se planteó el diagnóstico preoperatorio de cáncer. La sobrevida actuarial de los pacientes con $\mathrm{CMu}$ fue de $92 \%$ a cinco años.

Respecto de la localización, $74 \%$ de los $\mathrm{CMu}$ eran inaparentes y no fueron detectados macroscópicamente. En aquellos casos en que fue posible identificar lesiones sospechosas, éstas estaban localizadas predominantemente en el fondo y cuerpo (21\%) y sólo 4,3\% (9 casos) eran lesiones solevantadas o polipoideas. En 95\% (196 casos) se observó signos de inflamación crónica de la pared vesicular con lesiones de carácter proliferativo (hiperplasia de la mucosa, infiltración linfocitaria), lesiones metaplásicas (gástrica e intestinal), 0 bien, de tipo cicatricial (esclerosis vascular, fibrosis, atrofia). Todos los casos correspondieron a adenocarcinomas. En 96 casos se examinó histológicamente el ganglio cístico, en ningún caso se encontró tumor. En sólo 6 casos, en los bordes del tumor se encontraron focos adenomatosos en la mucosa adyacente. En los restantes 204 casos el mapeo sistematizado de la vesícula biliar no logró demostrar evidencias histológicas de un adenoma preexistente.

Gradiente morfológica displasia-carcinoma. En todos los grupos desde la colecistitis crónica, displasia hasta los distintos estadios de los CVB, se observó una clara gradiente entre la intensidad de la lesión y los promedios de edad de los pacientes (Figura 2). Las diferencias observadas entre los promedios de edad en el cual las lesiones fueron diagnosticadas fueron significativas en todos los grupos con un coeficiente de comelación de $\mathrm{R} 2=0,9899$ en los hombres y de (R2 $=0,9925$ ) en las mujeres (Figura 3).

La diferencia de los promedios de edad entre la colecistitis crónica y el CVB fue 10,3 años en el grupo general, 10,9 años en las mujeres y de 9,2 años en los hombres. Desde el punto de vista morfológico, el estudio de las características histológicas de vesículas biliares con displasia epitelial no asociada a cáncer, lesiones intraepiteliales atípicas asociadas a un CVB y el estudio sistematizado de la forma más precoz de CVB, nos permiten afirmar que los patrones histológicos que se observan en la displasia epitelial asociada o no a cáncer son congruentes con los observados en los CMu. Por el contrario, la ausencia de focos adenomatosos en los bordes de la forma más precoz de CVB, sustentan a la secuencia displasiacarcinoma como la vía más importante en el desarrollo de un CVB.

\section{DisCUSIÓN}

$\mathrm{Al}$ igual que otras neoplasias malignas epiteliales, el CVB es producto de la transformación del epitelio normal en lesiones consideradas como preneoplásicas y finalmente neoplásicas. En los órganos de fácil acceso es posible el seguimiento de estas lesiones, sin embargo, en la vesícula biliar 
esto no es posible, ya que una vez extraído el órgano el proceso se interrumpe. Escasa información existe respecto de la forma más precoz del CVB y que corresponde al $\mathrm{CMu}^{39-43}$. En países con alta incidencia de cáncer vesicular como Chile, India y Japón, el carcinoma mucoso puede llegar a representar hasta 15\% del total de cánceres vesiculares diagnosticados en colecistectomías $^{36}$.

Entre los dos modelos actualmente válidos para explicar la transformación maligna del epitelio vesicular displasia-carcinoma y adenoma-carcinoma ${ }^{25,28,44}$, los hallazgos morfológicos ${ }^{36,45}$ y genético-moleculares ${ }^{27,46-48}$, han demostrado que corresponden a dos eventos biológicos distintos e independientes. De acuerdo a nuestras observaciones, la secuencia displasia-carcinoma es la más verosímil desde el punto de vista morfológico $^{28,39,49,50}$. Por su parte, los adenomas son lesiones poco frecuentes en la vesícula biliar, su rol en la patogénesis del CVB es escasa.

La metaplasia es un hallazgo muy común en la mucosa vesicular crónicamente inflamada en sobre $50 \%$ de las vesículas biliares. Dos tipos de metaplasia son las que más frecuentemente se observan en la mucosa vesicular, la de tipo pilórica o gástrica $\mathrm{y}$ la de tipo intestinal ${ }^{10,30,51,52}$. Los CVB se asocian a la presencia de ambos tipos de metaplasias, especialmente la de tipo intestinal ${ }^{10,53}$. La secuencia sería metaplasia-displasia-CIS $25,52,54$. Algunos CVB presen-

\section{REFERENCIAS}

1. Lazcano-Ponce EC, Miquel JF, Munoz N, Herrero R, FerReCiO C, Wistuba II et al. Epidemiology and molecular pathology of gallbladder cancer. CA Cancer J Clin 2001; 51: 349-64.

2. PANDEy M. Risk factors for gallbladder cancer: a reappraisal. Eur J Cancer Prev 2003; 12: 15-24.

3. Andia ME, Hsing AW, Andreotti G, Ferreccio C. Geographic variation of gallbladder cancer mortality and risk factors in Chile: a population-based ecologic study. Int J Cancer 2008; 123: 1411-6.

4. SERRA I. ¿̇Ha disminuido la mortalidad por cáncer de la vesícula biliar en Chile? Rev Méd Chile 2001; 129: 1079-84. tarían un fenotipo "gástrico" con expresión de pepsinógenos I y II por parte de las células tumorales ${ }^{55,56}$. Aun cuando, la metaplasia intestinal y gástrica, parecen ser las lesiones previas a la displasia epitelial, no existen estudios concluyentes al respecto. Se ha demostrado inestabilidad microsatelital, $\mathrm{LOH}$ e inactivación de genes como p53, CDH1, p16 con mayor frecuencia en los focos de metaplasia intestinal en la mucosa asociada a cánceres que en la mucosa de colecistitis crónica ${ }^{47}$.

Otro elemento que sustenta este modelo de progresión, guarda relación con las edades de los pacientes a las cuales estas lesiones son diagnosticadas. Se observa una correlación casi exacta entre el aumento de la edad y la intensidad de las lesiones, hasta es posible sugerir una estimación del tiempo requerido en esta transformación ${ }^{28}$. De acuerdo a estos hallazgos el período de transformación desde la colecistitis crónica hasta el $\mathrm{CMu}$ sería de 10,9 años para la mujer y 9,2 años para el hombre. También se debe mencionar que el extrapolar estos hallazgos a la población con litiasis asintomática pudiese ser no necesariamente acertado.

Finalmente, es posible señalar que el CVB representa un modelo especial en la carcinogénesis humana que requiere mayor atención y del cual valiosa e importante información se pudiese obtener para el estudio y comprensión de los procesos neoplásicos en el ser humano.
5. Andia KM, GederLini GA, Ferreccio RC. Gallbladder cancer: trend and risk distribution in Chile. Rev Méd Chile 2006; 134: 565-74.

6. Wistuba, II, Gazdar AF. Gallbladder cancer: lessons from a rare tumour. Nat Rev Cancer 2004; 4: 695-706.

7. Misra S, Chaturvedi A, Misra NC, Sharma ID. Carcinoma of the gallbladder. Lancet Oncol 2003; 4: 167-76.

8. Kodama $\mathrm{K}$, NaKadaiRa $\mathrm{H}$, ENDOH $\mathrm{K}$, Yamamoto $\mathrm{M}$. Geographic clustering patterns in mortality from biliary tract cancer in Japan. Jpn J Cancer Res 1998; 89: 6-11.

9. Roa I, de Aretxabala X, Araya JC, Roa J. Preneoplastic lesions in gallbladder cancer. J Surg Oncol 2006; 93: 615-23.

10. Duarte I, Luanos O, Domke H, Harz C, Valdivieso V. 
Metaplasia and precursor lesions of gallbladder carcinoma. Frequency, distribution, and probability of detection in routine histologic samples. Cancer 1993; 72: 1878-84.

11. Lowenfeis AB, Maisonneuve P, Boyle P, Zatonski WA. Epidemiology of gallbladder cancer. Hepatogastroenterology 1999; 46: 1529-32.

12. Chow WH, Johansen C, Gridiey G, MeLiemkJaer L, OLSEN JH, FRAUMENI JF JR. Gallstones, cholecystectomy and risk of cancers of the liver, biliary tract and pancreas. Br J Cancer 1999; 79: 640-4.

13. Lowenfels AB, Walker AM, Althaus DP, Townsend G, DOMELOF L Gallstone growth, size, and risk of gallbladder cancer: an interracial study. Int J Epidemiol 1989; 18: 50-4.

14. Pandey M, Shukia VK. Lifestyle, parity, menstrual and reproductive factors and risk of gallbladder cancer. Eur J Cancer Prev 2003; 12: 269-72.

15. Lowenfels AB, Walker AM, Althaus DP, Townsend G, DOMELOF L. Gallstone growth, size, and risk of gallbladder study. Int J Epidemiol 1989; 18: 50-4.

16. Serra I, Calvo A, Maturana M, Medina E, Sharp A. Changing trends of gall-bladder cancer in Chile, a high-risk area. Int J Cancer 1990; 45: 376-7.

17. Chianale J, del Pino G, Nervi F. Increasing gallbladder cancer mortality rate during the last decade in Chile, a high-risk area. Int J Cancer 1990; 46: 1131-3.

18. Roa I, de Aretxabaia X, Morgan R, Molna R, Araya JC, RoA J et AL. Pólipos y adenomas de la vesícula biliar: características clínico-patológicas. Rev Méd Chile 2004; 132: 673-9.

19. KozuKa S, Tsubone N, Yasui A, Hachisuka K. Relation of adenoma to carcinoma in the gallbladder. Cancer 1982; 50: 2226-34.

20. Jung YS, Lee KJ, Kim H, Kim WH, Kim IG, Yoo BM eт AL. Risk factor for extrahepatic bile duct cancer in patients with anomalous pancreaticobiliary ductal union. Hepatogastroenterology 2004; 51: 946-9.

21. Matsumoto $Y$, FujII $H$, ItakuRa J, Matsuda M, NobuKawa B, SudA K. Recent advances in pancreaticobiliary maljunction. J Hepatobiliary Pancreat Surg 2002; 9: 45-54.

22. Donohue JH. Present status of the diagnosis and treatment of gallbladder carcinoma. J Hepatobiliary Pancreat Surg 2001; 8: 530-4.

23. SHAFFER EA. Epidemiology and risk factors for gallstone disease: has the paradigm changed in the 21st century? Curr Gastroenterol Rep 2005; 7: 132-40.

24. Roa I, de Aretxabala X, Araya JC, Viшaseca M, Roa J, GILDA IT ET AL. Elementos morfológicos pronósticos en el cáncer de la vesícula biliar. Rev Méd Chile 2002; 130: 387-95.

25. Albores-Saavedra J, Alcantra-Vázquez A, Cruz-Ortiz $\mathrm{H}$, HerRera-GoepFert R. The precursor lesions of invasive gallbladder carcinoma. Hyperplasia, atypical hyperplasia and carcinoma in situ. Cancer 1980; 45: 919-27.
26. Albores-Saavedra J, Vardaman CJ, Vuitch F. Nonneoplastic polypoid lesions and adenomas of the gallbladder. Pathol Annu 1993; 28 Pt 1: 145-77.

27. Wistuba, II, Miquel JF, Gazdar AF, Albores-Saavedra J. Gallbladder adenomas have molecular abnormalities different from those present in gallbladder carcinomas. Hum Pathol 1999; 30: 21-5.

28. Roa I, Araya JC, Viliaseca M, De Aretxabaia X, Riedemann P, ENDOH K et al. Preneoplastic lesions and gallbladder cancer: an estimate of the period required for progression. Gastroenterology 1996; 111: 232-6.

29. Wistuba, II, Sugio K, Hung J, Kishimoto Y, Virmani AK, ROA I ET AL. Allele-specific mutations involved in the pathogenesis of endemic gallbladder carcinoma in Chile. Cancer Res 1995; 55: 2511-5.

30. Laitio M. Histogenesis of epithelial neoplasms of human gallbladder I. Dysplasia. Pathol Res Pract 1983; 178: 51-6.

31. Smok G, Cervilia K, Bosch H, Csendes A. Lesiones precancerosas asociadas al carcinoma invasor de la vesícula biliar. Rev Méd Chile 1986; 114: 954-8.

32. OJeda VJ, ShilKin KB, Walters MN. Premalignant epithelial lesions of the gall bladder: a prospective study of 120 cholecystectomy specimens. Pathology 1985; 17: 451-4.

33. Mukhopadhyay S, Landas SK. Putative precursors of gallbladder dysplasia: a review of 400 routinely resected specimens. Arch Pathol Lab Med 2005; 129: 386-90.

34. Tsuchida A, Itoi T, Aoki T, Koyanagi Y. Carcinogenetic process in gallbladder mucosa with pancreaticobiliary maljunction (Review). Oncol Rep 2003; 10: 1693-9.

35. Matsubara T, Sakurai Y, Zhi LZ, Miura H, Ochiai M, FunABIKI T. K-ras and p53 gene mutations in noncancerous biliary lesions of patients with pancreaticobiliary maljunction. J Hepatobiliary Pancreat Surg 2002; 9: 312-21.

36. Roa I, Araya JC, Viliaseca M, Roa J, de Aretxabala X, IBACACHE G. Gallbladder cancer in a high risk area: morphological features and spread patterns. Hepatogastroenterology 1999; 46: 1540-6.

37. Gallbladder. In: American Joint Committee on Cancer: AJCC Cancer Staging Manual. Philadelphia, Pa: Lippincott-Raven Publishers 1997; $5^{\text {th }}$ ed.: 103-108.

38. Gallbladder. In American Joint Committee on Cancer: AJCC Cancer Staging Manual. 6th ed. New York, NY: Springer. 2002; 139-44.

39. Roa I, de Aretxabala X, Araya JC, Viliaseca M, Roa J, GuZmán P. Carcinoma incipiente de la vesícula biliar. Estudio clínico, patológico y pronóstico en 196 casos. Rev Méd Chile 2001; 129: 1113-20.

40. De AretXabala X, Roa I, Burgos L. Gallbladder cancer, management of early tumors. Hepatogastroenterology 1999; 46: 1547-51.

41. TsuchiYA Y. Early carcinoma of the gallbladder: macroscopic features and US findings. Radiology 1991; 179: 171-5. 
42. Latrio M. Early carcinoma of the gallbladder. Beitr Pathol 1976; 158: 159-72.

43. Wagholikar GD, Behari A, Krishnani N, Kumar A, Sikora SS, SAXENA R ET AL. Early gallbladder cancer. J Am Coll Surg 2002; 194: 137-41.

44. Albores-Saavedra J, Henson DE, Sobin LH. The WHO Histological Classification of Tumors of the Gallbladder and Extrahepatic Bile Ducts. A commentary on the second edition. Cancer 1992; 70: 410-4.

45. Roa I GP, Ibacache G, Araya J, Villaseca M, de Aretxabala X, Roa J, Muñoz S. Cáncer de la vesícula biliar en colecistectomías por litiasis. Rev Esp Patol 2004; 37: 279-85.

46. Yanagisama N, Mikami T, Saegusa M, Okayasu I. More frequent beta-catenin exon 3 mutations in in carcinomas indicate different lineages. Cancer Res 2001; 61: 19-22.

47. Roa JC, Roa I, Correa P, Vo Q, Araya JC, Vilaseca M ET AL. Microsatellite instability in preneoplastic and neoplastic lesions of the gallbladder. J Gastroenterol 2005; 40: 79-86.

48. Wistuba, II, Albores-SaAvedra J. Genetic abnormalities involved in the pathogenesis of gallbladder carcinoma. J Hepatobiliary Pancreat Surg 1999; 6: 237-44.

49. Roa I, Araya JC, Wistuba I, de AretXabala X. Cáncer vesicular: consideraciones anatómicas y anatomopatológicas. Rev Méd Chile 1990; 118: 572-9.
50. Roa I, Araya JC, Wistuba I, Viliaseca M, de Aretxabala X, BUSEL D ET AL. Lesiones epiteliales asociadas al cáncer carcinoma de la vesícula biliar. Estudio sistematizado de 32 casos. Rev Méd Chile 1993; 121: 21-9.

51. Kozuka S, Kurashina M, Tsubone M, Hachisuka K, YASUI A. Significance of intestinal metaplasia for the evolution of cancer in the biliary tract. Cancer 1984; 54: 2277-85.

52. Dowlng GP, KELY JK. The histogenesis of adenocarcinoma of the gallbladder. Cancer 1986; 58: 1702-8.

53. Yamagiwa H. Mucosal dysplasia of gallbladder: isolated and adjacent lesions to carcinoma. Jpn J Cancer Res 1989; 80: 238-43.

54. Albores-Saavedra J, Nadjo M, Henson D, ZiegelsWeIsSman J, Mones J. Intestinal metaplasia of the gallblader: A morphologic and immunocytochemical study. Hum Pathol 1986; 17: 614-20.

55. Roa I, Araya JC, Shiraishi T, Yatani R, Wistuba I, VILLASECA M ET AL. Demostración inmunohistoquímica de pepsinógenos I and II en el cáncer de la vesícula biliar. Rev Méd Chile 1992; 120: 1351-8.

56. Tatematsu M, Ichinose M, Miki K, Tatematsu K, KishikaWA H, Iто N. Gastric phenotypic expression in human gallbladder cancers revealed by pepsinogen immunohistochemistry and mucin histochemistry. Virchows Arch A Pathol Anat Histopathol 1988; 413: 25-32. 